\title{
Variability in foot contact patterns in independent walking in infants
}

\author{
Carina Price*, Stewart Morrision and Christopher Nester \\ School of Health Sciences, University of Salford, UK \\ School of Health Sciences, University of Brighton, UK
}

\section{Introduction}

Independent walking in infants can be defined as the ability to walk 5-10 steps without support. Plantar pressures in independent infant walking exist in literature (Bertsch et al., 2004; Müller et al., 2012), but data has been captured in environments which impact on the infant's typical gait patterns. For example walking in straight lines for set numbers of steps being dictated and encouraged by researchers. This is likely to lower the gait variability, for example by reducing the variability in progression angles, standardising the direction they are facing and increasing the number of steps taken in one walking bout (trial). Thus producing data with low external validity in terms of the loading on the plantar surface of the infant foot in real world environments. Additionally, mean data for groups of infants is presented in existing literature, with no measure of the within-infant variability. This is despite consensus that reducing variability is key in development of experienced walking (Bertsch et al., 2004; Hallemans et al., 2004).

\section{Purpose of the study}

The purpose of this work was to quantify plantar pressures and contact areas (and variability thereof) in natural independent walking in infants.

\section{Methods}

Data was collected on four infants walking independently in an infant lab (8-12 weeks of independent walking, 3 Female). An Emed-xl platform (100 Hz; Novel, Munich) with a resolution of 4 sensors per $\mathrm{cm}^{2}$ was positioned in the centre of a $2.0 * 2.3 \mathrm{~m}$ crèche scene. Two video cameras $(50 \mathrm{~Hz})$ in corners of the area were synchronized with the pressure platform. The environment included foam flooring, imagery, toys and cubes akin to a crèche (Figure 1).

Infants were accompanied by a parent in the cordoned (with baby gates) area for approximately 5 minutes while data was collected continuously. Infants were encouraged to move by the researcher and parent from standardised positions away from the platform. Otherwise the infant was left to play and move around as they wished. Steps were manually extracted and analysed using Novel Multimask software. Steps analysed were dynamic steps where the infant was ambulating and foot contact was not on the platform border (confirmed using video), irrespective of velocity or direction of progression. Total foot contact area and peak pressures were evaluated; medians, inter-quartile ranges (IQR) and ranges are presented for comparison. No statistical comparison was undertaken at this stage. 


\section{Results}

19-35 steps met the criteria for inclusion from each infant (example 6 steps from one infant Figure 2).

Mean total foot contact area was $45.1 \mathrm{~cm}^{2}$ (range $42.6-46.4 \mathrm{~cm}^{2}$ ) and IQRs for each infant were $9.9-13.1 \%$ of the magnitude of the median total contact area $\left(42.5-46.4 \mathrm{~cm}^{2}\right)$. The difference between the minimum and maximum contact patterns across steps for each infant were 22.8$38.7 \%$ of the median total contact area for each infant; an average difference of $14.8 \mathrm{~cm}^{2}$.

Median peak pressures across the total foot and all steps for each infant ranged from 105$165 \mathrm{kPa}$ with IQRs of $35-92.5 \mathrm{kPa}$. Total foot peak pressure values ranged from $70-330 \mathrm{kPa}$ across all steps and all infants.

\section{Discussion and conclusion}

Capturing "typical" foot loading patterns during independent walking in infants walking denotes a highly variable contact pressure on the plantar surface of the foot. Mean pressure and contact area for the infants were similar to those calculated using the mean of 3-5 steps in a 2 step protocol (Müller, et al., 2012) and the mean of 5 steps collected in the middle of straight line walking (Bertsch et al., 2004). However, our work demonstrates that there is a large range of loading on the plantar foot within individuals around these mean values.

The infant who produced the maximum peak pressure value of $330 \mathrm{kPa}$ moved faster in the lab and demonstrated the lowest contact area IQR. Supporting that variability of measures is relevant to quantify walking development maturity.

This research is limited by no observational analysis of infants in a familiar environment e.g. at home. Additionally, capturing variability in a prescribed methodology would have aided a direct comparison and a true reflection of the threat to external validity current methodologies incorporate. Our sample size is an evident limitation, which will be increased in continuing work.

\section{References}

Bertsch, C. et al., (2004). Gait \& Posture 19 (3), 235-242.

Müller, S. et al. (2012). Gait \& Posture 35 (3), 389-394.

Hallemans et al., (2003). Foot Ankle Int. 24 (5), 444-453. 


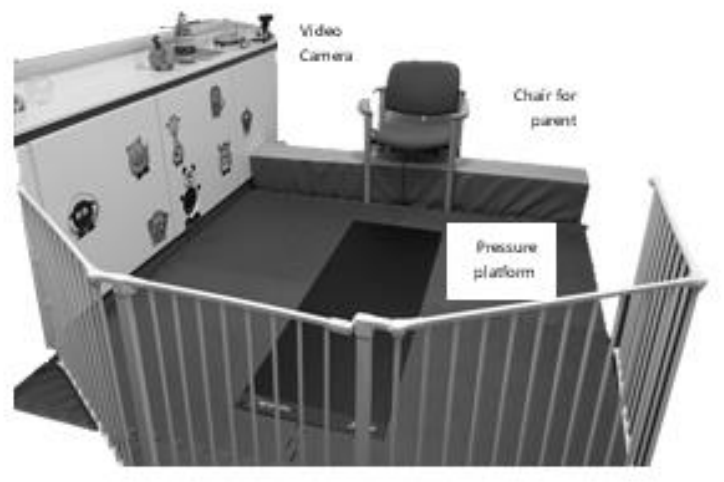

Figure 1. Laboratory environment for pressure data collection

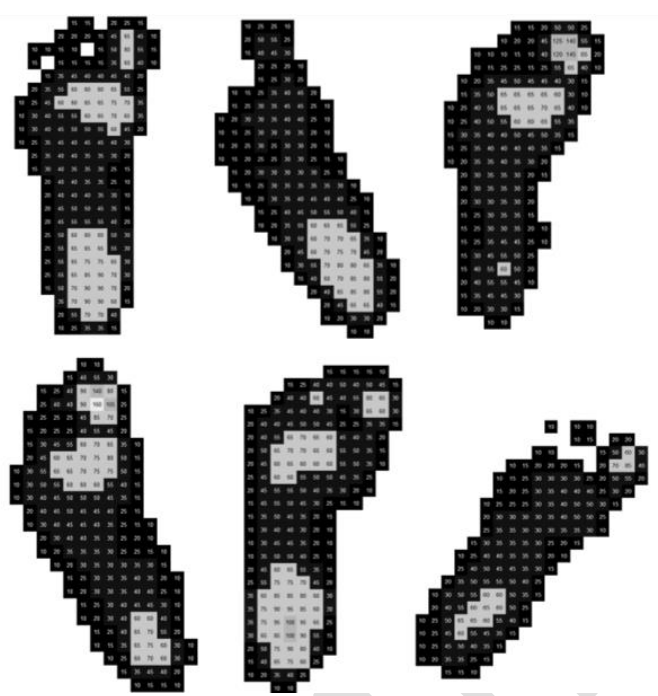

Figure 2. Representative 6 peak pressure steps plantar pressure of the total 24 analysed from infant 2. 